\title{
Fractional cable equation for general geometry, a model of axons with swellings and anomalous diffusion
}

\author{
Erick J. López-Sánchez ${ }^{(1) *}$, Juan M. Romero ${ }^{(2)}{ }^{\dagger}$ Huitzilin Yépez-Martínez ${ }^{(3)}{ }^{\ddagger}$ \\ (1) Posgrado en Ciencias Naturales e Ingeniería, \\ Universidad Autónoma Metropolitana, Cuajimalpa. \\ Vasco de Quiroga 4871, Santa Fe Cuajimalpa, D. F. 05300 México. \\ ${ }^{(2)}$ Departamento de Matemáticas Aplicadas y Sistemas, \\ Universidad Autónoma Metropolitana-Cuajimalpa, \\ México, D.F 05300, México \\ (3) Universidad Autónoma de la Ciudad de México, \\ Prolongación San Isidro 151, San Lorenzo Tezonco, Iztapalapa, \\ Ciudad de México 09790, México.
}

\begin{abstract}
Different experimental studies have reported anomalous diffusion in brain tissues and notably this anomalous diffusion is expressed through fractional derivatives. Axons are important to understand neurodegenerative diseases such as multiple sclerosis, Alzheimer's disease and Parkinson's disease. Indeed, abnormal accumulation of proteins and organelles in axons is a hallmark feature of these diseases. The diffusion in the axons can become to anomalous as a result from this abnormality. In this case the voltage propagation in axons is affected. Another hallmark feature of different neurodegenerative diseases is given by discrete swellings along the axon. In order to model the voltage propagation in axons with anomalous diffusion and swellings, in this paper we propose a fractional cable equation for general geometry. This generalized equation depends on fractional parameters and geometric quantities such as the curvature and torsion of the cable. For a cable with a constant radius we show that the voltage decreases when the fractional effect increases. In cables with swellings we find that when the fractional effect or the swelling radius increase, the voltage decreases. A similar behavior is obtained when the number of swellings and the fractional effect increase. Moreover, we find that when the radius swelling (or the number of swellings) and the fractional effect increase at the same time, the voltage dramatically decreases.
\end{abstract}

\section{Introduction}

In biological organisms there are hydrogen atoms in abundance, particularly in water and fat. These atoms allow to study biological organisms with non invasive techniques, such as Magnetic Resonance Imaging (MRI). Indeed, using a magnetic field and the Zeeman effect, the diffusion process of water molecules in biological tissues can be mapped [1, 2]. Thus, using these techniques it is possible to study some physiological properties of biological tissues, for example water molecules diffusion patterns can reveal microscopic details about tissue architecture and can reflect interactions with many obstacles, such as macromolecules, fibers, and membranes

\footnotetext{
*lsej@unam.mx

†jromero@correo.cua.uam.mx

†huitzilin.yepez.martinez@uacm.edu.mx
} 
[1, 2, 3, 4. Notably, in neuroscience water diffusion can provide information about white matter integrity, fiber density, uniformity of nerve fiber direction, axonal membranes and cytoskeleton properties, etc [3, 4]. In fact, water diffusion has been used to detect and characterize different neurodegenerative diseases [5]. For instance, using Diffusion Tensor Imaging (DTI), altered diffusion has been detected in white matter of subjects with multiple sclerosis [6, 7, 8, Also, using DTI, white matter alterations were found in the corpus callosum of subjects with Huntinton's disease [9]. Moreover, using DTI, relevant white matter abnormalities were found in Alzheimer's disease [10, 11]. In addition, using MRI, hippocampal atrophy has been detected in Parkinson's disease [12]. Even more, different experimental studies have shown that water diffusion in some tissues can not be described by a Gaussian model, but as an anomalous diffusion expressed through fractional calculus [13, 14, 15, 16, 17, 18], in particular in brain tumours [19, 20, 21] and dendrites [22]. Furthermore, some authors have suggested that the anomalous diffusion parameters might play a role in the diagnosis of brain diseases [23]. It is worth mentioning that in different neurodegenerative diseases are reported abnormal accumulations of proteins and organelles which generate disruption axonal transport [24, 25, 26]. Additionally, different theoretical studies support the claim that anomalous diffusion appears in a heterogeneous medium [27, 28, 29]. For these reasons, diffusion and anomalous diffusion in brain tissues are relevant in the study of the brain physiology. Other studies about anomalous diffusion in cellular system can be seen in [30, 31, 32, 33.

Another essential aspect to understand how the brain works is given by its electrical activity. Thus, in order to obtain a reasonable model of the brain, it is important to know how the voltage propagates in brain tissues with anomalous diffusion. In particular, due that axons are crucial in neuron-to-neuron communications and those can be described as cables, we should know how the voltage propagates in a cable with anomalous diffusion. In this respect, to study the voltage $V(x, t)$ in a straight cylindrical cable with a circular cross-section of constant diameter $d_{0}$ and anomalous diffusion, recently some authors have proposed a fractional cable equation as follows [34, 35]

$$
c_{M} \frac{\partial V(x, t)}{\partial t}=\beta_{\nu} D_{\nu t}\left(\frac{d_{0}}{4 r_{L}} \frac{\partial^{2} V(x, t)}{\partial x^{2}}-i_{\text {ion }}\right),
$$

where $c_{M}$ denotes the specific membrane capacitance, $r_{L}$ denotes the longitudinal resistance and $i_{\text {ion }}$ is the ionic current per unit area into and out of the cable,

$$
D_{\nu t}=\frac{\partial^{1-\nu}}{\partial t^{1-\nu}}, \quad \nu=\text { constant }, \quad 0 \leq \nu \leq 1
$$

is the Riemann-Liouville fractional operator [36] and $\beta_{\nu}$ is a constant with $(\text { time })^{1-\nu}$ dimensions. The passive cable case, namely when $i_{i o n}=V / r_{M}$ (where $r_{M}$ is the specific membrane resistance) was used to study electrodiffusion of ions in nerve cells [34, 35].

Additionally, there are different physiological phenomena where the geometry is important. For instance, axons with non trivial geometry are important to understand some neurodegenerative diseases, indeed discrete swellings along the axons appear in neurodegenerative diseases such as Alzheimer's disease, Parkinson's disease, HIV-associated dementia and multiple sclerosis. In fact, axons with a diameter of approximately $1 \mu \mathrm{m}$ with a swelling with diameter of approximately $5 \mu \mathrm{m}$, are reported in Parkinson's disease [37]. In addition, axons with a diameter of approximately $4 \mu \mathrm{m}$ with a swelling with a diameter of approximately $60 \mu \mathrm{m}$ are reported in multiple sclerosis [38]; axons with a diameter of approximately $1.5 \mu \mathrm{m}$ with a swelling train, where the swelling diameter varies between $4 \mu \mathrm{m}$ and $10 \mu \mathrm{m}$, are reported in Alzheimer's disease [41, 39, 40]; axons with a diameter of approximately $6 \mu \mathrm{m}$ and swellings with a diameter of approximately $43 \mu \mathrm{m}$ are reported in HIV-associated dementia [42, 43, 44, 45, 46]. Other sizes of the axonal swellings can be see in [47, 48]. Some theoretical studies on cables with non cylindrical geometry can be seen in [49, 50, 51, 52, 53, 54, 55, 56]. Because the equation (1) only describes axons with cylindrical geometry, therefore, in order to study the voltage propagation in a cable with non trivial geometry and anomalous diffusion, this equation should be 
generalized.

In this paper, we study the voltage propagation in a cable with anomalous diffusion and non trivial geometry. For this purpose, we introduce a fractional cable equation for a general geometry. This generalized equation depends on fractional parameters $\beta_{\nu}$ and $\nu$ and geometric quantities such as the curvature and torsion of the cable. For different cable geometries, we show that with regard to model a system where the voltage decreases, we should suppose that $\beta_{\nu}$ increases when $\nu$ decreases. For a straight cylinder with a constant radius we show that the voltage depends on neither the curvature nor the torsion of the cable and it decreases when $\beta_{\nu}$ increases and $\nu$ decreases. In addition, cables with swellings are studied. In these last cable geometries we find that when the swelling radius increases or $\beta_{\nu}$ increases and $\nu$ decreases, the voltage decreases dramatically.

This paper is organized as follows: in the section 2 we propose a fractional cable equation with a general geometry; in the section 3 we analyse some general properties of the generalized fractional cable equation; in the section 4 we consider the cylindrical cable with a constant radius; in the section 5 we study cable with swellings. Finally, in the section 6 a summary is given.

\section{Fractional cable equation in a general geometry}

It is well known that to study the geometric properties of a three dimensional curve $\vec{\gamma}$ the arc length parameter

$$
s=\int_{0}^{x} \sqrt{\frac{d \vec{\gamma}(\zeta)}{d \zeta} \cdot \frac{d \vec{\gamma}(\zeta)}{d \zeta}} d \zeta
$$

is a friendly parameter. Indeed, using the arc length parameter (3) we can construct the vectors of the Frenet-Serret frame [57]

$$
\frac{d \vec{\gamma}(s)}{d s}=\hat{T}, \quad \hat{N}=\frac{\frac{d \hat{T}}{d s}}{\left|\frac{d \hat{T}}{d s}\right|}, \quad \hat{B}=\hat{T} \times \hat{N}
$$

where $\hat{T}$ is the unit vector tangent, $\hat{N}$ is the normal unit vector and $\hat{B}$ is the binormal unit vector to the curve. Furthermore, using the arc length and the Frenet-Serret frame, the Frenet-Serret formulas can be obtained as follow [57]

$$
\frac{d \hat{T}}{d s}=\kappa \hat{N}, \quad \frac{d \hat{N}}{d s}=-\kappa \hat{T}+\tau \hat{B}, \quad \frac{d \hat{B}}{d s}=-\tau \hat{N}
$$

where $\kappa, \tau$ are the curvature and torsion of the curve $\vec{\gamma}$, respectively.

We can employ the Frenet-Serret frame to construct a cable model. Actually, we can propose a general cable as the region bounded by the following surface

$$
\vec{\Sigma}(\theta, s)=\vec{\gamma}(s)+f_{1}(\theta, s) \hat{N}(s)+f_{2}(\theta, s) \hat{B}(s),
$$

where $\theta$ is an angular variable. Notice that employing the angular coordinate $\theta$, the functions $f_{1}(\theta, s), f_{2}(\theta, s)$ and the vectors $\hat{N}(s), \hat{B}(s)$ we are constructing the cable over the curve $\vec{\gamma}(s)$. In Fig. 1 we can see a representation of the surface (6). For instance, a cable with a deformed circular cross-section, where the radius $R$ depends on the angle $\theta$, can be described by the surface (6) where

$$
f_{1}(\theta, s)=R(\theta, s) \cos \theta, \quad f_{2}(\theta, s)=R(\theta, s) \sin \theta .
$$

Notice that in this case the cross-section area is given by

$$
a(s)=\frac{1}{2} \int_{0}^{2 \pi} R^{2}(\theta, s) d \theta .
$$


Some geometric quantities as the area of a surface can be written in terms of the first fundamental form, which is constructed with the inner product on the tangent space of a surface as follows [57]

$$
g=\left(\begin{array}{cc}
E & F \\
F & G
\end{array}\right)
$$

where

$$
\begin{aligned}
& E=\frac{\partial \vec{\Sigma}(\theta, s)}{\partial s} \cdot \frac{\partial \vec{\Sigma}(\theta, s)}{\partial s} \\
& G=\frac{\partial \vec{\Sigma}(\theta, s)}{\partial \theta} \cdot \frac{\partial \vec{\Sigma}(\theta, s)}{\partial \theta} \\
& F=\frac{\partial \vec{\Sigma}(\theta, s)}{\partial s} \cdot \frac{\partial \vec{\Sigma}(s, \theta)}{\partial \theta} .
\end{aligned}
$$

Now, let us remember that the curvature $\kappa(s)$ at a point $P$ of the curve $\vec{\gamma}(s)$ is defined as the inverse of the radius of the osculating circle at $P$, see Ref. [58. Then, if the radius of the osculating circle is small, the surface (6) describes a cable with a big curvature. In addition, notice that when the radius of the osculating circle is smaller than the radius of the cable, the cable surface touches itself. In the literature there are not reported axons with big curvature, then in this paper we suppose that at each point of the curve $\vec{\gamma}(s)$ the radius of the osculating circle is larger than the radius of the cable. Namely, at each point of the curve $\vec{\gamma}(s)$, we suppose that the curvature $\kappa(s)$ is smaller than $R^{-1}(s)$ and the following inequality

$$
\kappa(s) R(s)<1
$$

is satisfied.

The axon geometry is important for diverse physiological processes, such as the voltage propagation. In this respect, according to Ref. [56], when the cable geometry is given by (6) the cable equation is

$$
\frac{\partial V(s, t)}{\partial t}=\frac{1}{r_{L} c_{M} \int_{0}^{2 \pi} d \theta \sqrt{\operatorname{det} g(\theta, s)}} \frac{\partial}{\partial s}\left(a(s) \frac{\partial V(s, t)}{\partial s}\right)-\frac{i_{i o n}}{c_{M}},
$$

where $a(s)$ is the cable cross-section and

$$
\begin{aligned}
\sqrt{\operatorname{det} g(\theta, s)}= & {\left[R^{2}(\theta, s)\left(\frac{\partial R(\theta, s)}{\partial s}-\tau \frac{\partial R(\theta, s)}{\partial \theta}\right)^{2}\right.} \\
& \left.+(1-\kappa(s) R(\theta, s) \cos \theta)^{2}\left(R^{2}(\theta, s)+\left(\frac{\partial R(\theta, s)}{\partial \theta}\right)^{2}\right)\right]^{\frac{1}{2}}
\end{aligned}
$$

Notice that the equation (14) depends on geometric quantities as the curvature $\kappa$ and torsion $\tau$ of the cable.

Then, in order to study the voltage propagation in a cable with general geometry and anomalous diffusion, we can employ the equations (1) and (14) to propose a generalized fractional cable equation as follows

$$
\frac{\partial V(s, t)}{\partial t}=\beta_{\nu} D_{\nu t}\left[\frac{1}{r_{L} c_{M} \int_{0}^{2 \pi} d \theta \sqrt{\operatorname{det} g(\theta, s)}} \frac{\partial}{\partial s}\left(a(s) \frac{\partial V(s, t)}{\partial s}\right)-\frac{i_{\text {ion }}}{c_{M}}\right],
$$

where

$$
D_{\nu t}=\frac{\partial^{1-\nu}}{\partial t^{1-\nu}}, \quad \nu=\text { constant }, \quad 0 \leq \nu \leq 1
$$


is the Riemann-Liouville fractional operator [36] and $\beta_{\nu}$ is a constant with $(\text { time })^{1-\nu}$ dimensions. The voltage in an infinite cable has to satisfy the Dirichlet boundary condition and a finite cable has to satisfy the Neumann boundary condition [59, 60], then the solutions of the equation (16) should satisfy these boundary conditions.

In the general case, $i_{\text {ion }}$ depends on the voltage and the equation (14) is a non linear differential equation. However, in the passive cable model we can take

$$
i_{\text {ion }}=\frac{V(s, t)}{r_{M}}
$$

Therefore, the fractional cable equation for the passive cable model with the geometry given by $(6)$ is

$$
\frac{\partial V(s, t)}{\partial t}=\beta_{\nu} D_{\nu t}\left[\frac{1}{r_{L} c_{M} \int_{0}^{2 \pi} d \theta \sqrt{\operatorname{det} g(\theta, s)}} \frac{\partial}{\partial s}\left(a(s) \frac{\partial V(s, t)}{\partial s}\right)-\frac{V(s, t)}{r_{M} c_{M}}\right] .
$$

In the next sections we will study some solutions of this equation.

\section{A qualitative analysis}

For a non trivial geometry, to find solutions of the equation $(19)$ is a difficult task. However, let us provide a qualitative analysis of this equation. In this respect, we propose the following voltage

$$
V(s, t)=\mathcal{T}(t) X(s)
$$

In this case the equation $(19)$ implies the following equations

$$
\begin{aligned}
\frac{1}{r_{L} c_{M} \int_{0}^{2 \pi} d \theta \sqrt{\operatorname{det} g(\theta, s)} \frac{\partial}{\partial s}\left(a(s) \frac{\partial X(s)}{\partial s}\right)-\frac{X(s)}{r_{M} c_{M}}}=-\lambda X(s) \\
\frac{\partial \mathcal{T}(t)}{\partial t}=-\lambda \beta_{\nu} D_{\nu t} \mathcal{T}(t),
\end{aligned}
$$

where $\lambda$ is a constant.

Moreover, if we take

$$
X(s)=\frac{\psi(s)}{\sqrt{a(s)}},
$$

the spacial equation $(21)$ can be written as

$$
-\frac{\partial^{2} \psi(s)}{\partial s^{2}}+U(s) \psi(s)=0
$$

where

$$
U(s)=\frac{r_{L} c_{M} \int_{0}^{2 \pi} d \theta \sqrt{\operatorname{det} g(\theta, s)}}{a(s)}\left(\lambda-\frac{1}{r_{M} c_{M}}\right)-\frac{1}{2}\left(\frac{\left(\frac{d a(s)}{d s}\right)^{2}}{2 a^{2}(s)}-\frac{\frac{d^{2} a(s)}{d s^{2}}}{a(s)}\right) .
$$

Observe that in this last equation the parameters $\nu$ and $\beta_{\nu}$ do not appear. In fact, this spacial equation is the same spacial equation which appears in the non fractional case [56]. As well, observe that the parameter $\lambda$ does not depend on $\nu$ neither on $\beta_{\nu}$. 
Furthermore, the Laplace transform of the temporal equation 22 implies

$$
\tilde{\mathcal{T}}_{\nu}(\zeta)=\mathcal{T}(0) \frac{\zeta^{\nu-1}}{\left(\zeta^{\nu}+\beta_{\nu} \lambda\right)}
$$

where $\tilde{\mathcal{T}}_{\nu}(\zeta)$ is the Laplace transform of the function $\mathcal{T}_{\nu}(t)$. The inverse Laplace transform of the function 26 is given by 36

$$
\mathcal{T}_{\nu}(t)=\mathcal{T}(0) E_{\nu, 1}\left(-\beta_{\nu} \lambda t^{\nu}\right)
$$

where

$$
E_{\nu, \Lambda}(z)=\sum_{n \geq 0} \frac{z^{n}}{\Gamma(\nu n+\Lambda)}
$$

is the Mittag-Leffler type function [36]. Notice that if $\nu=1$ we obtain the usual solution

$$
\mathcal{T}_{1}(t)=\mathcal{T}(0) e^{-\lambda t}
$$

In addition, if $\nu=0$ we obtain

$$
\mathcal{T}_{0}(t)=\mathcal{T}(0) \sum_{n \geq 0}\left(-\lambda \beta_{0}\right)^{n}
$$

which is a constant. We can observe that the equation (30) only makes sense if

$$
\beta_{0} \lambda<1
$$

in this case we get

$$
\mathcal{T}_{0}(t)=\frac{\mathcal{T}(0)}{1+\beta_{0} \lambda}
$$

In the section IV, we can see that, for realistic parameter for cylindrical cable, $\lambda$ is the order of $10^{-3}(\mathrm{sec})^{-1}$. Then, the inequality (31) is satisfied if $\beta_{0}$ is smaller than $10^{3} \mathrm{sec}$.

Notice that the strongest fractional effect is obtained when $\nu$ is close to zero and in this case the function $\mathcal{T}_{\nu}(t)$ is close to the constant (32), which decreases when $\beta_{0}$ increases. Then, in order to model a system where the voltage decreases, we should take $\beta_{0}$ bigger than one, but satisfying the inequality (31). If we take $\beta_{0}$ close to zero, the voltage does not decrease.

Now, remember that the usual case is obtained with $\nu=1$ and $\beta_{1}=1$. Additionally, when $\nu$ is close to zero, $\beta_{\nu}$ should reach its maximum value, in fact $\beta_{0}$ should be bigger than one. Thus, for a system where the voltage decreases, we can suppose that $\beta_{\nu}$ increases when $\nu$ decreases.

Fig. 2 shows the function (27) for different $\beta_{\nu}$ and $\nu$ values. In this we can see that the function (27) decreases when $\beta_{\nu}$ increases and $\nu$ decreases.

Then, using the functions (27) and (23), we found the following voltage

$$
V(s, t)=\frac{\mathcal{T}(0)}{\sqrt{a(s)}} E_{\nu, 1}\left(-\beta_{\nu} \lambda t^{\nu}\right) \psi(s),
$$

where the function $\psi(s)$ satisfies the equation (24). From the equation (33) we can see that when the cable cross-sectional area $a(s)$ increases the voltage decreases. As well, when $\beta_{\nu}$ increases and $\nu$ decreases the voltage decreases.

Therefore, this qualitative analysis suggests that in an axon with transport or geometrical defects the voltage decreases. 


\section{Cylindrical cable with constant radius}

When the sectional area is a constant, that is $R(s)=R_{0}=$ constant, the equation (15) does not depend on the curvature neither on the torsion of the cable. Indeed, in this case the equation (19) becomes

$$
\frac{\partial V(s, t)}{\partial t}=\beta_{\nu} D_{\nu t}\left(\frac{R_{0}}{2 c_{M} r_{L}} \frac{\partial^{2} V(s, t)}{\partial s^{2}}-\frac{V(s, t)}{r_{M} c_{M}}\right)
$$

which is equivalent to the fractional cable equation for a straight cylindrical cable (1). However, observe that the equation (34) depends on the arc length parameter (3) instead of the lab frame coordinate. This shows that the natural variables for the voltage are given by geometric quantities of the cable.

For these geometries, in the finite cable case, the solution of the equation (34) is given by

$$
V(s, t)=\sum_{n \geq 0} b_{n} E_{\nu, 1}\left[-\beta_{\nu}\left(\frac{n \pi R_{0}}{2 c_{M} r_{L} l}+\frac{1}{r_{M} c_{M}}\right) t^{\nu}\right] \cos n \pi \frac{s}{l},
$$

where $l$ is the length of the cable and $E_{\nu, \Lambda}(z)$ is the Mittag-Leffler function (28).

Fig. 3 shows the fractional cable equation solution for a cylindrical cable for different $\nu$ and $\beta_{\nu}$ values and the initial condition

$$
V(s, 0)=A\left(1+\cos \left(\frac{s \pi}{l}\right)\right)
$$

where $A=0.05 \mathrm{mV} \cdot \mathrm{cm}^{\frac{1}{2}}$ and $l=0.13 \mathrm{~cm}$. In this figure, we can see that the voltage decreases when $\beta_{\nu}$ increases and $\nu$ decreases. Then, anomalous diffusion implies that voltage decreases.

To obtain an exact solution of the equation (34) is a difficult task for an arbitrary initial condition. Then, in order to study this case we employ a numerical method. For integer derivatives, spacial and temporal, we use a second order finite differences method. Moreover, for the temporal fractional derivatives we use a second order scheme taken from the Fractional Integration Toolbox [61]. The mesh size was chosen as follows: first, we begin with 1024 points along the s-axis and 100 points at the time. These numbers were increasing until the difference between two successive solutions was almost null. The number of spatial and temporal points used in the simulations are shown in Table 1. The system is solved using the Gauss-Seidel iterative method, with a tolerance of $10^{-10}$. In addition, we impose the Neumann boundary conditions [59]

$$
\frac{\partial V\left(s_{0}, t\right)}{\partial s}=\frac{\partial V\left(s_{n_{s}}, t\right)}{\partial s}=0 .
$$

Fig. 4 shows the numerical solution of the equation (34) for a cylindrical cable for different $\nu$ and $\beta_{\nu}$ values and the initial condition (36). This figure shows that the numerical solution is close to the analytical solution.

Table 1: Number of points, $n_{s}$ and $n_{t}$, and number of times of the refinement.

\begin{tabular}{|l|l|l|}
\hline Refinement & $n_{s}$ & $n_{t}$ \\
\hline First time & 1024 & 100 \\
\hline Second time & 2048 & 500 \\
\hline Third time & 4096 & 2000 \\
\hline
\end{tabular}


A more realistic initial condition is given by the function

$$
V(s, 0)=\frac{A}{\sqrt{2 \pi \sigma}} e^{-\frac{s^{2}}{2 \sigma^{2}}},
$$

where $A=0.00128 \mathrm{mV} \cdot \mathrm{cm}^{\frac{1}{2}}$ and $\sigma=0.004 \mathrm{~cm}$. Fig. 5 shows the numerical solution of equation (34) for different $\nu$ and $\beta_{\nu}$ values and the initial condition (38). The numerical values of $\nu$ and $\beta_{\nu}$ used in this simulation are shown in Table 2 .

Notice that both the exact and the numerical solutions of the equation (34) provide a voltage which decreases when $\beta_{\nu}$ increases and $\nu$ decreases. Then, anomalous diffusion implies that voltage decreases. It is worth mentioning that some experimental studies show that in brain tumours there is anomalous diffusion [19, 20, 21]. As well, in different neurodegenerative diseases disruption axonal transport are reported [24, 25, 26]. In these cases, the fractional cable equation implies that the voltage decreases.

In the next section we will study the equation 19 with non constant radius.

\section{$5 \quad$ Cables with swellings}

It can be shown that when a cable has vanished curvature and the cable radius is given by

$$
R(s)=R_{0}\left(1+\alpha_{1} \sin \alpha_{2} s\right)
$$

or

$$
R(s)=R_{0}\left(1+\alpha_{1} \sin ^{2} \alpha_{2} s\right)
$$

the spatial equation (24) is similar to the spatial equation for the straight cylindrical cable. For this reason, the voltage in a cable with radius (39) or (40) is similar to the voltage in the straight cylindrical cable [56]. Hence, in the fractional case, when a cable has vanished curvature and the radius is given by (39) or 40 ) the voltage will be similar to voltage (35).

\subsection{Circular cross-section}

The cable equation (19) is hard to solve for a general cable geometry. However, for some cases this equation can be simplified. For instance, when the cable has a circular cross-section, namely when $R(\theta, s)=R(s)$, the equation $(19)$ does not depend on the torsion of the cable $\tau$ and becomes

$$
\frac{\partial V(s, t)}{\partial t}=\beta_{\nu} D_{\nu t}\left(\frac{\pi \frac{\partial}{\partial s}\left(R^{2}(s) \frac{\partial V(s, t)}{\partial s}\right)}{r_{L} c_{M} R(s) \int_{0}^{2 \pi} d \theta \sqrt{(1-\kappa(s) R(s) \cos \theta)^{2}+\left(\frac{d R(s)}{d s}\right)^{2}}}-\frac{V(s, t)}{r_{M} c_{M}}\right) .
$$

In the following subsections we will study cables which model axons with swellings.

Table 2: Numerical values of $\nu$ and $\beta_{\nu}$.

\begin{tabular}{|l|l|}
\hline$\nu$ & $\beta_{\nu}$ \\
\hline 1 & 1 \\
\hline 0.9 & $1.5(\mathrm{sec})^{0.1}$ \\
\hline 0.7 & $4(\mathrm{sec})^{0.3}$ \\
\hline 0.5 & $16(\mathrm{sec})^{0.5}$ \\
\hline 0.3 & $37(\mathrm{sec})^{0.7}$ \\
\hline
\end{tabular}




\subsection{Cable with Gaussian swelling}

In different neurodegenerative diseases, focal axonal swellings are found such as the Fig. 6(a), see for example [38]. This geometry can be modelled with a cable with radius

$$
R(s)=R_{0}\left(1+\alpha_{1} e^{-\alpha_{2}\left(s-\alpha_{3}\right)^{2}}\right) .
$$

For this cable geometry, if the initial condition is given by (38), the numerical solution of the equation (41) can be seen in the Fig. 6(b), (c) and (d). In these figures we can observe that the voltage decreases faster than the voltage of the cylindrical cable. In addition, notice that for this cable geometry when $\beta_{\nu}$ increases and $\nu$ decreases the voltage of the cable decreases faster than when $\beta_{1}=1$ and $\nu=1$. Then, in an axon with a swelling and anomalous diffusion the voltage strongly decreases.

\subsection{Cable with Gaussian swellings}

In this section we study the numerical solution for the equation (41) for a cable with the radius

$$
R(s)=R_{0}\left(1+\alpha_{1} e^{-\alpha_{2} s^{2}}+\alpha_{1} e^{-\alpha_{2}\left(s-\alpha_{3}\right)^{2}}+\alpha_{1} e^{-\alpha_{2}\left(s-2 \alpha_{3}\right)^{2}}+\alpha_{1} e^{-\alpha_{2}\left(s-3 \alpha_{3}\right)^{2}}\right)
$$

and with the initial condition (38).

A cable with this geometry can be seen in the figure 7(a). It is worth mentioning that axons with this geometry have been reported in different studies [48]. The numerical solution of the equation (41) for this cable radius can be observed in Fig. 7(b), (c) and (d). In this case the voltage decreases faster than the voltage of the cylindrical cable. Moreover, when $\beta_{\nu}$ increases and $\nu$ decreases, the voltage decreases faster than non fractional voltage $\left(\beta_{1}=\nu=1\right)$. In addition, we can see that the voltage in a cable with this geometry decreases faster than the voltage in a cable with the geometry (42).

\subsection{Amorphous swelling}

A more realistic model for an axon with swelling is given by Fig. 8(a). This geometry can be described by a cable with an amorphous swelling with radius

$$
R(\theta, s)=R_{0}\left(1+\alpha_{1} e^{-\alpha_{2}\left(s-\alpha_{3}\right)^{2}}+\alpha_{4} \sin \theta \cos \alpha_{5} s\right) .
$$

The numerical solution of the voltage for this cable can be seen in Fig. 8(b), (c) and (d). This voltage is different from the voltage for the cable with radius (42). Then, geometric inhomogeneities affects the voltage propagation in a cable.

\section{Summary}

In different neurodegenerative diseases such as multiple sclerosis, Alzheimer's disease and Parkinson's disease are reported abnormal accumulations of proteins and organelles which generate disruption axonal transport. In addition, recently different experimental studies have found anomalous diffusion in brain tissues. Notably this diffusion is expressed through fractional calculus. Another hallmark feature of some neurodegenerative diseases is given by axonal discrete swellings along the axons.

In order to study the voltage propagation in a cable with both of these hallmark features of the neurodegenerative diseases, we proposed a fractional cable equation with non trivial geometry. This equation depends on geometric quantities such as the curvature and torsion of the cable, as well as the fractional parameters $\beta_{\nu}$ and $\nu$. It is worth mentioning that the parameter $\beta_{\nu}$ depends on $\nu$. In this respect, we showed that with regard to model a system where the voltage decreases, we should suppose that $\beta_{\nu}$ increases when $\nu$ decreases. Furthermore, in this 
new cable equation the strongest fractional effect is obtained when $\nu$ is close to zero.

For a straight cylinder with a constant radius we showed that the voltage decreases when the fractional effect increases. Notice that in this case the cable does not have swellings. Then, if there is an abnormal accumulation of proteins and organelles in an axon, the diffusion can be hindered and become to anomalous diffusion. In this case our results suggest that the voltage decreases in axons. Indeed, when the fractional effect is strong, the voltage may be blocked.

In addition, cables with swellings and anomalous diffusion were studied. Regarding this, we studied cable geometries similar to some axons reported in the literature. For all these cable geometries, we found that when the fractional effect increases, the voltage decreases. Furthermore, we found that the voltage dramatically decreases when the cable has a big swelling or has many swellings. Then, these results also suggested that in axons with swellings and abnormal accumulations of proteins and organelles the voltage decreases and may be blocked.

How axonal transport defects and deformed geometry of axons are related each other is an important problem. In order to study this problem, in our model the geometry quantities of the cable should be related with the parameters $\nu$ and $\beta_{\nu}$. In a future work the above problem and the active case, where non linear interactions play an important role, will be studied.

\section{Acknowledgments}

This work was supported in part by conacyt-sep 47510318 (J.M.R.). We are grateful to referees for providing valuable comments.

\section{References}

[1] I. L. Pykett, NMR Imaging in Medicine, Scientific American 246, 78 (1986).

[2] I. L. Pikett, J. H Newhouse, F. S. Buonanno, T. J. Brady, M. R. Goldman, J. P. Kistler, and G. M. Pohost, Principles of nuclear magnetic resonance imaging, Radiology 143, 157 (1981).

[3] J.-D. Tournier, S. Mori, and A. Leemans, Diffusion tensor imaging and beyond, Magnetic Resonance in Medicine 65, 1532 (2011).

[4] C. Beaulieu, The basis of anisotropic water diffusion in the nervous system - a technical review, NMR Biomed., 15, 435 (2002).

[5] J. Goveas, L. O’ Dwyer, M. Mascalchi, M. Cosottini, S. Diciotti, S. De Santis, L. Passamonti, C.Tessa, N. Toschi, and M. Giannelli, Diffusion-MRI in neurodegenerative disorders, Magnetic Resonance Imaging 33, 853 (2015).

[6] S. K. Song, S. W. Sun, M. J. Ramsbottom, C. Chang, J. Russell, and A. H. Cross, Dysmyelination revealed through MRI as increased radial (but unchanged axial) diffusion of water, NeuroImage 17, 1429 (2002).

[7] D. J. Werring, C. A. Clark, G. J. Barker, A. J. Thompson, and D. H. Miller, Diffusion tensor imaging of lesions and normal-appearing white matter in multiple sclerosis, Neurology 521626 (1999).

[8] M. Filippi, M. Cercignani, M. Inglese, M. A. Horsfield, and G. Comi, Diffusion tensor magnetic resonance imaging in multiple sclerosis, Neurology 56304 (2001).

[9] H. D. Rosas, D. S. Tuch, N. D. Hevelone, A. K. Zaleta, M. Vangel, S. M. Hersch, and D. H. Salat, Diffusion tensor imaging in presymptomatic and early Huntington's disease: Selective white matter pathology and its relationship to clinical measures, Movement Disorders 21, 1317 (2006). 
[10] S. J. Choi, K. O. Lim, I. Monteiro, and B. Reisberg, Diffusion tensor imaging of frontal white matter microstructure in early Alzheimers disease: a preliminary study, Journal of Geriatric Psychiatry and Neurology 18, 12 (2005).

[11] N. H. Stricker, B. C. Schweinsburg, L. Delano-Woodd, C. E. Wierengad, K. J. Bangen, K. Y.Haaland, L. R. Frank, D.P.Salmon, and M.W.Bondi, Decreased white matter integrity in late-myelinating fiber pathways in Alzheimer's disease supports retrogenesis, NeuroImage 4510 (2009).

[12] M. P. Laakso, K. Partanen,P. Riekkinen, M. Lehtovirta, E. L. Helkala, M. Hallikainen, T. Hanninen, P. Vainio, and H. Soininen, Hippocampal volumes in Alzheimer's disease, Parkinson's disease with and without dementia, and in vascular dementia: an MRI study, Neurology 46, 678 (1996).

[13] Y. Bai, Y. Lin, J. Tian, D. Shi, J. Cheng, E. M. Haacke, X. Hong, B. Ma, J. Zhou, and M. Wang, Grading of gliomas by using monoexponential, biexponential, and stretched exponential diffusion-weighted MR imaging and diffusion kurtosis MR imaging, Radiology 278, 496 (2016).

[14] M. M. Karaman, Y. Sui, H. Wang, R. L. Magin, Y. Li, and X. J. Zhou, Differentiating low- and high-grade pediatric brain tumors using a continuous-time random-walk diffusion model at high b-values, Magnetic Resonance in Medecine 76 4, 1149 (2016).

[15] B. Xu, L. Su, Z. Wang, Y. Fan, G. Gong, W. Zhu, P. Gao, and J.-H. Gao, Anomalous diffusion in cerebral glioma assessed using a fractional motion model, Magnetic Resonance in Medecine (2017). DOI: 10.1002/mrm.26581.

[16] R. L. Magin, O. Abdullah, D. Baleanu, and X. J. Zhoua, Anomalous diffusion expressed through fractional order differential operators in the Bloch-Torrey equation, Journal of Magnetic Resonance 190, 255 (2008).

[17] K. M. Bennett, K. M. Schmainda, R. T. Bennett, D. B. Rowe, H. Lu, and J. S. Hyde, Characterization of continuously distributed cortical water diffusion rates with a stretchedexponential model, Magnetic Resonance in Medicine 50, 727 (2003).

[18] K. M. Bennett, J. S. Hyde, and K. M. Schmainda, Water diffusion heterogeneity index in the human brain is insensitive to the orientation of applied magnetic field gradients, Magnetic Resonance in Medicine 56, 235 (2006).

[19] B. Xu, L. Su, Z. Wang, Y. Fan, G. Gong, W. Zhu, P. Gao, and J.-H. Gao, Anomalous diffusion in cerebral glioma assessed using a fractional motion model, Magnetic Resonance in Medicine, (2017) doi:10.1002/mrm.26581.

[20] M. M. Karaman, H. Wang, Y. Sui, H. H. Engelhard, Y. Li, and X. J. Zhou, A fractional motion diffusion model for grading pediatric brain tumors, NeuroImagen: Clinical 12, 707 (2016).

[21] X.-J. Zhou, Q. Gao, O. Abdullah, and R. L. Magin, Studies of anomalous diffusion in the human brain using fractional order calculus, Magnetic Resonance in Medicine 63, 562 (2010).

[22] F. Santamaria, S. Wils, E. De Schutter, and G. J. Augustine, Anomalous diffusion in Purkinje cell dendrites caused by spines, Neuron 52, 365 (2006).

[23] S. Qin, F. Liu, I. W. Turner, Q. Yu, Q. Yang, and V. Vegh, Characterization of anomalous relaxation using the time-fractional Bloch equation and multiple echo $T_{2}^{*}$-weighted magnetic resonance imaging at 7 T, Magnetic Resonance in Medicine 77, 1485 (2017).

[24] E. Chevalier-Larsen and E. L. F. Holzbaur, Axonal transport and neurodegenerative disease, Biochimica et Biophysica Acta - Molecular Basis of Disease 1762, 1094 (2006). 
[25] K. J. De Vos, A. J. Grierson, S. Ackerley, and C. C. J. Miller, Role of axonal transport in neurodegenerative diseases, Annu. Rev. Neurosci. 31, 151 (2008).

[26] S. Millecamps and J.-P. Julien, Axonal transport deficits and neurodegenerative diseases, Nature Reviews Neuroscience 14, 161 (2013).

[27] R. Metzler and J. Klafter, The random walk's guide to anomalous diffusion: a fractional dynamics approach, Phys. Rep. 339, 1 (2000).

[28] D. S. Novikov, E. Fieremans, J. H. Jensen, and J. A. Helpern, Random walks with barriers, Nature Physics 7, 508 (2011).

[29] M. Palombo, A. Gabrielli, V. D. P. Servedio, G. Ruocco, and S. Capuani, Structural disorder and anomalous diffusion in random packing of spheres, Scientific Reports 3, 2631 (2013).

[30] J. M. Haugh, Analysis of reaction-diffusion systems with anomalous subdiffusion, Biophysical Journal 97, 435 (2009).

[31] M. J. Saxon, A biological interpretation of transient anomalous subdiffusion. I. Qualitative model, Biophysical Journal 92, 1178 (2007).

[32] M. Weiss, M. Elsner, F. Kartberg, and T. Nilsson, Anomalous subdiffusion is a measure for cytoplasmic crowding in living cells, Biophysical Journal 87, 3518 (2004).

[33] M. J. Saxon, Anomalous subdiffusion in fluorescence photobleaching recovery: a Monte Carlo study, Biophysical Journal 81, 2226 (2001).

[34] B. I. Henry, T. A. M. Langlands, and S. L. Wearne, Fractional cable models for spiny neuronal dendrites, Phys. Rev. Lett. 100, 128103 (2008).

[35] T. A. M. Langlands, B. I. Henry, and S. L. Wearne, Fractional cable equation models for anomalous electrodiffusion in nerve cells: infinite domain solutions, Journal of Mathematical Biology 59, 761 (2009).

[36] L. Debmath and D. Bhatta, Integral Transforms ans Their Applications 2nd ed. (Chapman and Hall/CRC, NewYork, 2007).

[37] J. E. Galvin, K. Uryu, V. M.-Y. Lee, and J. Q. Trojanowski, Axon pathology in Parkinson' $s$ disease and Lewy body dementia hippocampus contains $\alpha-, \beta-$, and $\gamma$-synuclein, Proc. Natl. Acad. Sci. USA 96, 13450 (1999).

[38] B. D. Trapp, J. Peterson, R. M. Ransohoff, R. Rudick, S. Mörk, and L. Bö, Axonal transection in the lesions of multiple sclerosis, New Engl. J. Med. 338, 278 (1998).

[39] V. E. Johnson, W. Stewart, and D. H. Smith, Axonal pathology in traumatic brain injury, Exp. Neurol. 246, 35 (2013).

[40] D. Krstic and I. Knuesel, Deciphering the mechanism underlying late-onset Alzheimer disease, Nat. Rev. Neurosci. 9, 25 (2013).

[41] H. Xie, J. Guan, L. A. Borrelli, J. Xu, A. Serrano-Pozo, and B. J. Bacskai, Mitochondrial alterations near amyloid plaques in an Alzheimer's disease mouse model, J. Neurosci. 33, 17042 (2013).

[42] H. Budka, G. Costanzi, S. Cristina, A. Leehi, C. Parravicini, R. Trabattoni, and L. Vago, Brain pathology induced by infection with the human immunodeficiency virus (HIV), Acta Neuropathologica 75, 185 (1987).

[43] F. Gray, F. Chrétien, A. V. Vallat-Decouvelaere, and F. Scaravilli, The Changing Pattern of HIV Neuropathology in the HAART Era, J. Neuropathol. Exp. Neurol. 62, 429 (2003). 
[44] M. Kaul, G. A. Garden, and S. A. Lipton, Pathways to neuronal injury and apoptosis in HIV-associated dementia, Nature 410, 988 (2001).

[45] F. Raja, F. E. Sherriff, C. S. Morris, L. R. Bridges, and M. M. Esiri, Cerebral white matter damage in HIV infection demonstrated using $\beta$-amyloid precursor protein immunoreactivity, Acta Neuropathol. 93, 184 (1997).

[46] R. Ellis, D. Langford, and E. Masliah, HIV and antiretroviral therapy in therain: neuronal injury and repair, Nat. Rev. Neurosci. 8, 33 (2007).

[47] M. H. Magdesian, F. S. Sanchez, M. Lopez, P. Thostrup, N. Durisic, W. Belkaid, D. Liazoghli, P. Grütter, and D.R. Colman, Atomic force microscopy reveals important differences in axonal resistance to injury, Biophys. J. 103, 405 (2012).

[48] G. M. G. Shepherd and K. M. Harris, Three-dimensional structure and composition of $C A 3 \rightarrow C A 1$ axons in rat hippocampal slices: Implications for presynaptic connectivity and compartmentalization, J. Neurosci. 18, 8300 (1998).

[49] H. Anwar, C. J. Roome, H. Nedelescu, W. Chen, B. Kuhn, and E. De Schutter, Dendritic diameters affect the spatial variability of intracellular calcium dynamics in computer models, Front. Cell. Neurosci. 8, 168 (2014).

[50] P. Vetter, A. Roth, and M. Hausser, Propagation of action potentials in dendrites depends on dendritic morphology, Neurophysiol 85, 926 (2001).

[51] A. D. Bird and H. Cuntz, Optimal current transfer in dendrites, PLoS Comput Biol. 12, e1004897 (2016).

[52] E. R. Kandel, J. H. Schwartz, and T. N. Jessell, Principles of Neural Science (McGraw-Hill, New York, 2000).

[53] J. C. Fiala and K. M. Harris, Dendrite structure, in Dendrites, edited by G. Stuart, N. Spruston, and M. Häusser (Oxford University Press, Oxford, 1999), p.1.

[54] R. R. Poznanski, Modelling the electrotonic structure of starburst amacrine cells in the rabbit retina: A functional interpretation of dendritic morphology, Bull. Math. Biol. 54, 905 (1992).

[55] P. D. Maia, M.A. Hemphill, B. Zehnder, C. Zhang, K. K. Parker, and J. N. Kutz, Diagnostic tools for evaluating the impact of Focal Axonal Swellings arising in neurodegenerative diseases and/or traumatic brain injury, Journal of Neuroscience Methods 253, 233 (2015).

[56] E. López-Sánchez and J. M. Romero, Cable equation for general geometry, Phys. Rev. E. 95, 022403 (2017).

[57] M. P. Do Carmo, Differential Geometry of Curves and Surfaces (Dover, NewYork, 2016).

[58] M. Spivak, A Comprehensive Introduction to Differential Geometry Vol. II (Publish or Perish, Houston 1975).

[59] G. B. Ermentrout and D. H. Terman, Mathematical Foundation of Neuroscience (Springer, London, 2010).

[60] P. C. Bressloff, Waves in Neural Media: From Single Neurons to Neural Fields (Springer, London, 2014).

[61] T. M. Marinov, N. Ramirez, and F. Santamaria, Fractional Integration Toolbox, Fractional Calculus and Applied Analysis, 16, 670 (2013). 


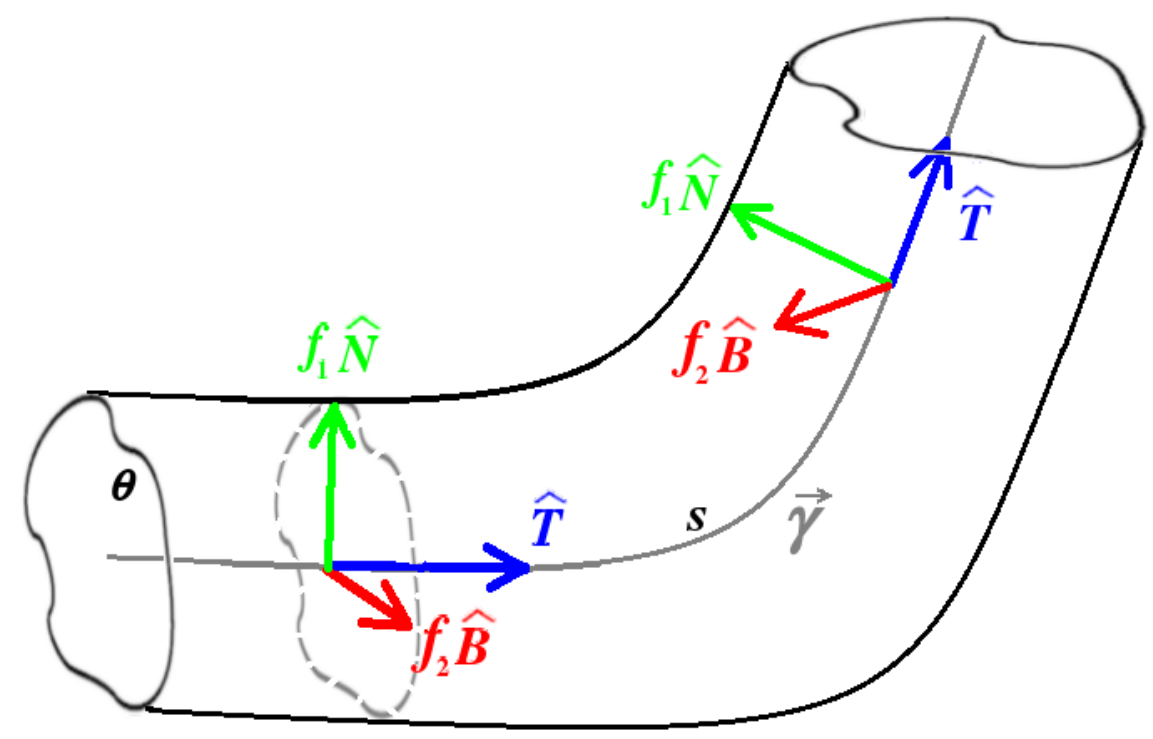

Figure 1: Cable with general geometry. The vectors $\hat{T}, \hat{N}, \hat{B}$ are shown in two different points on the curve $\vec{\gamma}$.

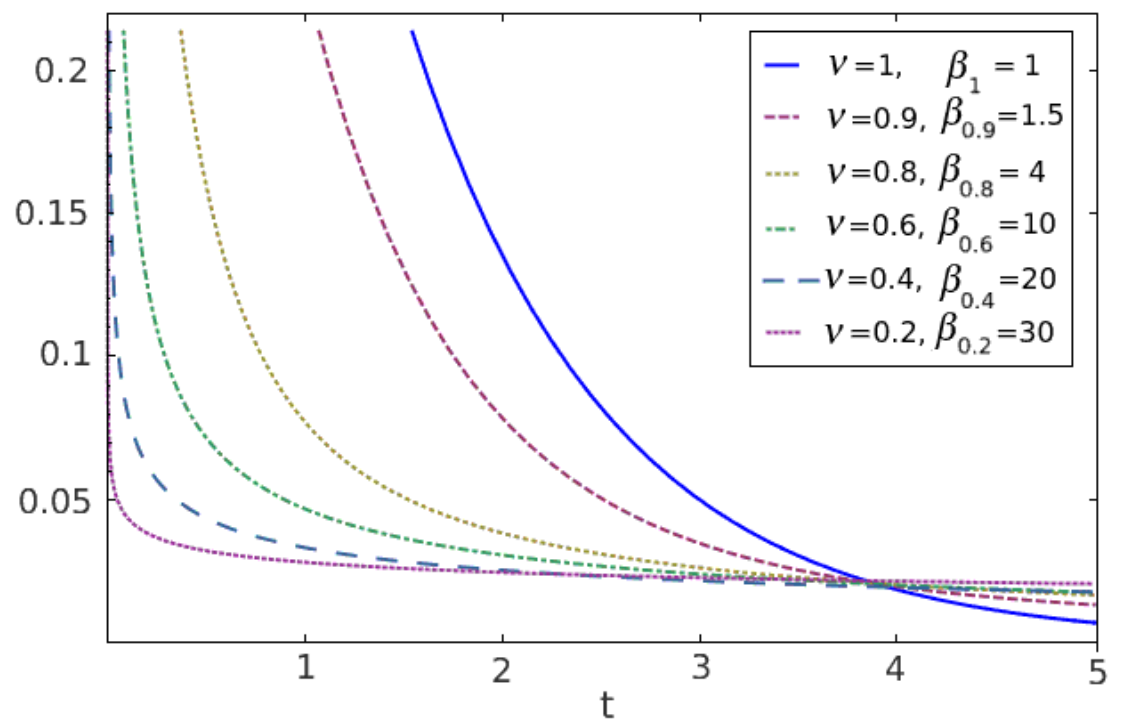

Figure 2: Mittag-Leffler with different values of $\beta_{\nu}$ and $\nu$.
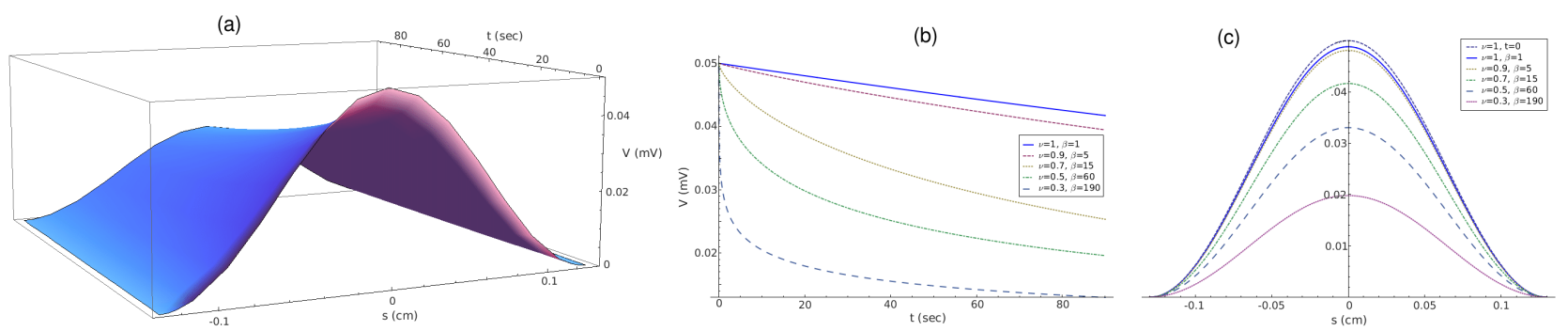

Figure 3: (a) Voltage for the cylindrical cable, with $\nu=0.7$ and $\beta_{0.7}=15(\mathrm{sec})^{0.3}$. (b) Voltage vs $t$ in $s=0$ with different values of $\beta_{\nu}$ and $\nu$. (c) Voltage vs $s$ at time $t=12 \mathrm{sec}$ for different values of $\beta_{\nu}$ and $\nu$. Parameter values used for simulations correspond to realistic dendritic parameters as in [54]: $c_{M}=1 \mathrm{mF} / \mathrm{cm}^{2}, r_{M}=3000 \Omega \mathrm{cm}^{2}, r_{L}=100 \Omega \mathrm{cm}, R_{0}=10^{-4} \mathrm{~cm}$. The initial condition is given by $(36)$. 
(a)

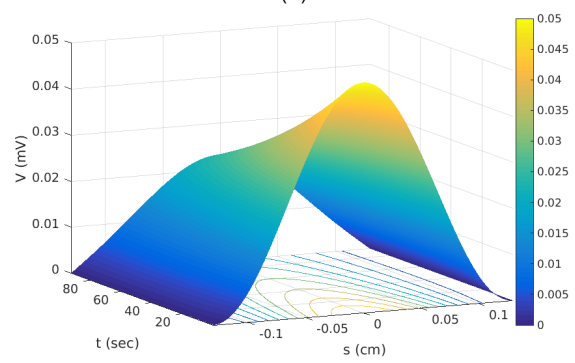

(b)

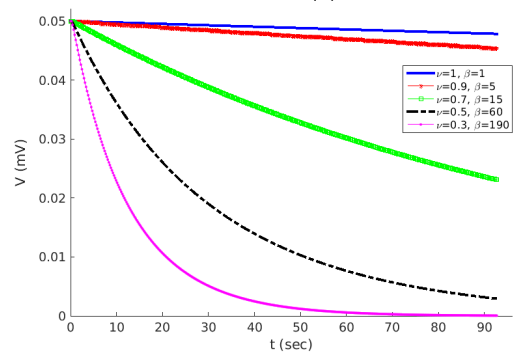

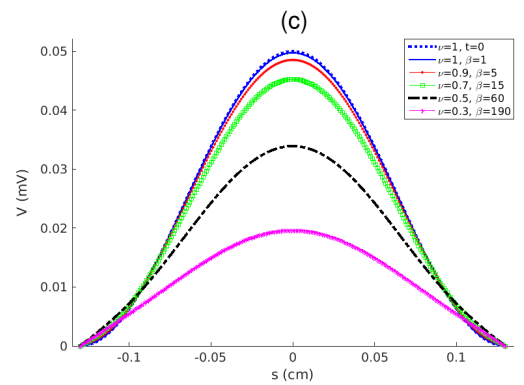

Figure 4: (a) Voltage for the cylindrical cable, with $\nu=0.7$ and $\beta_{0.7}=15$ (sec $)^{0.3}$. (b) Voltage vs $t$ in $s=0$ with different values of $\beta_{\nu}$ and $\nu$. (c) Voltage vs $s$ at time $t=12 \mathrm{sec}$ for different values of $\beta_{\nu}$ and $\nu$. Parameter values used for simulations correspond to realistic dendritic parameters as in [54]: $c_{M}=1 \mathrm{mF} / \mathrm{cm}^{2}, r_{M}=3000 \Omega \mathrm{cm}^{2}, r_{L}=100 \Omega \mathrm{cm}, R_{0}=10^{-4} \mathrm{~cm}$. The initial condition is given by (36).

(a)

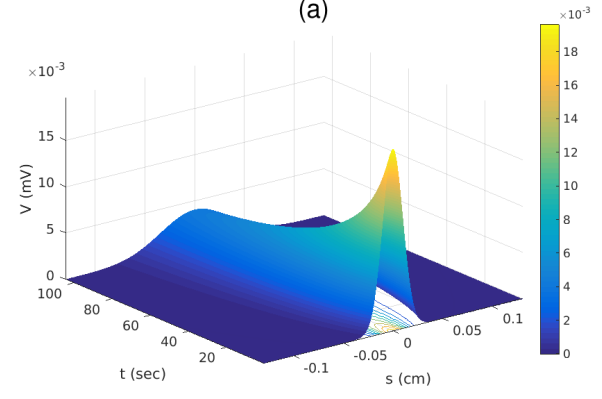

(b)

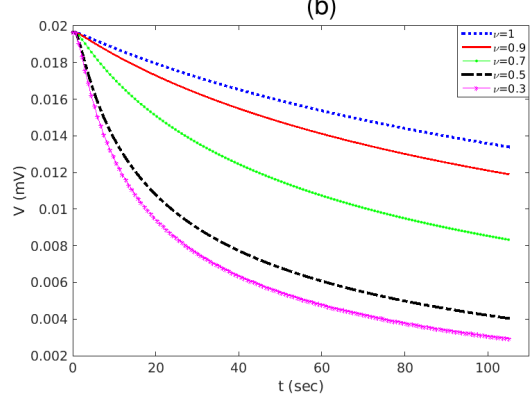

(c)

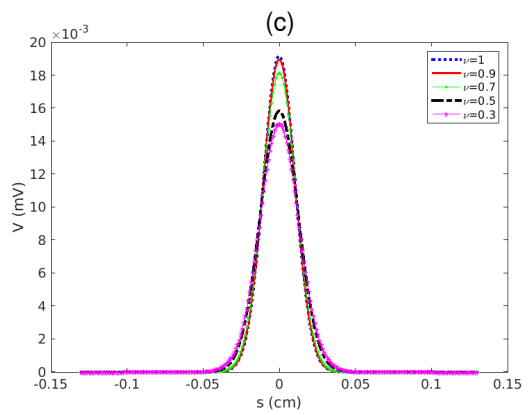

Figure 5: (a) Voltage for the cylindrical cable, with $\nu=0.5$ and $\beta_{0.5}=16(\mathrm{sec})^{0.5}$. (b) Voltage vs $t$ in $s=0$ with different values of $\beta_{\nu}$ and $\nu$. (c) Voltage vs $s$ at time $t=7 \mathrm{sec}$ for different values of $\beta_{\nu}$ and $\nu$. Parameter values used for simulations correspond to realistic dendritic parameters as in [54]: $c_{M}=1 \mathrm{mF} / \mathrm{cm}^{2}, r_{M}=3000 \Omega \mathrm{cm}^{2}, r_{L}=100 \Omega \mathrm{cm}, R_{0}=10^{-4} \mathrm{~cm}$. The initial condition is given by $(38)$. 
(a)

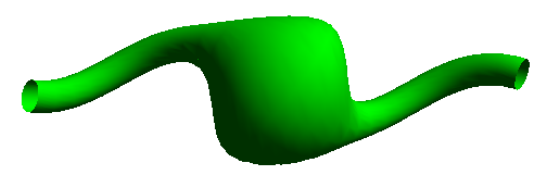

(b)
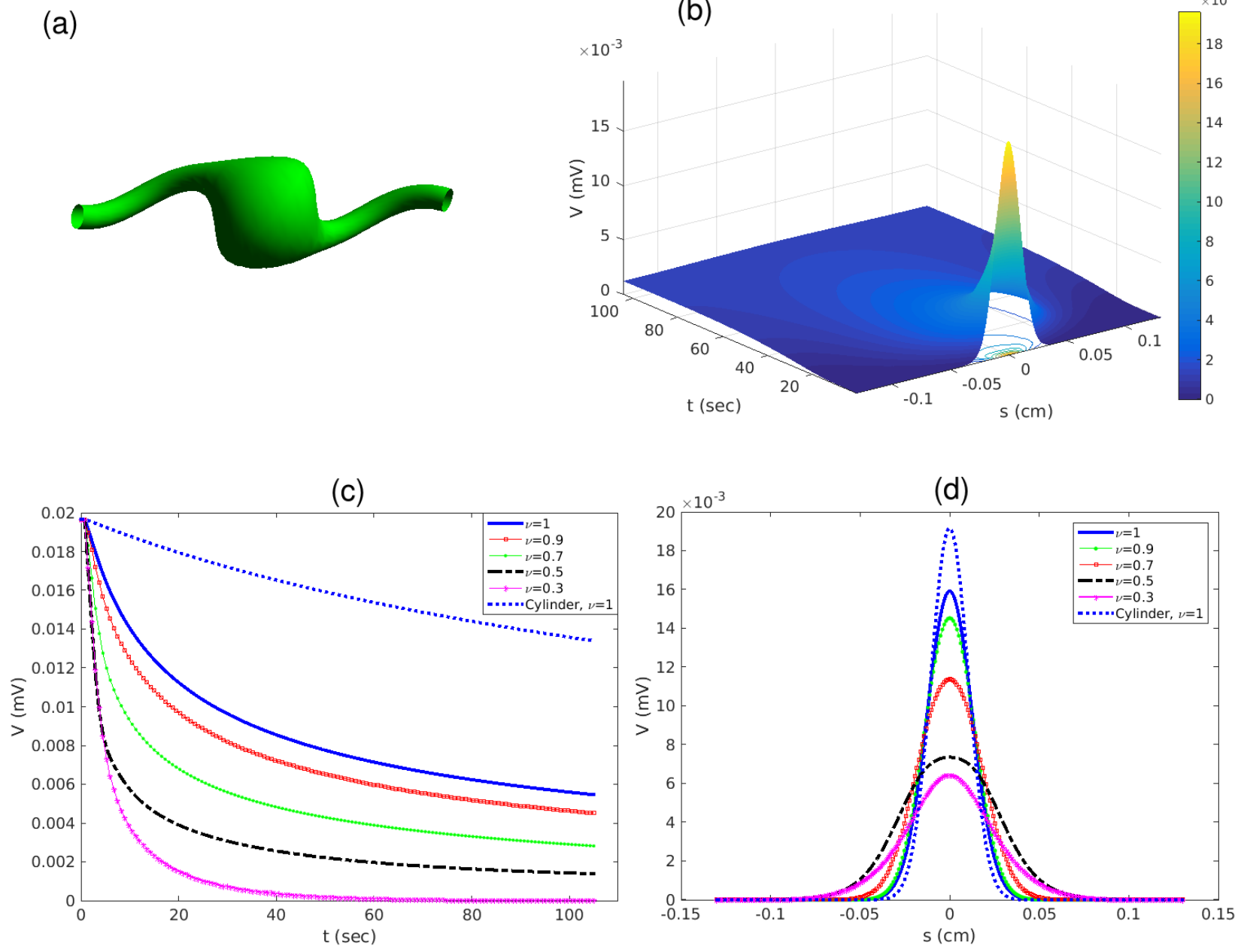

(d)

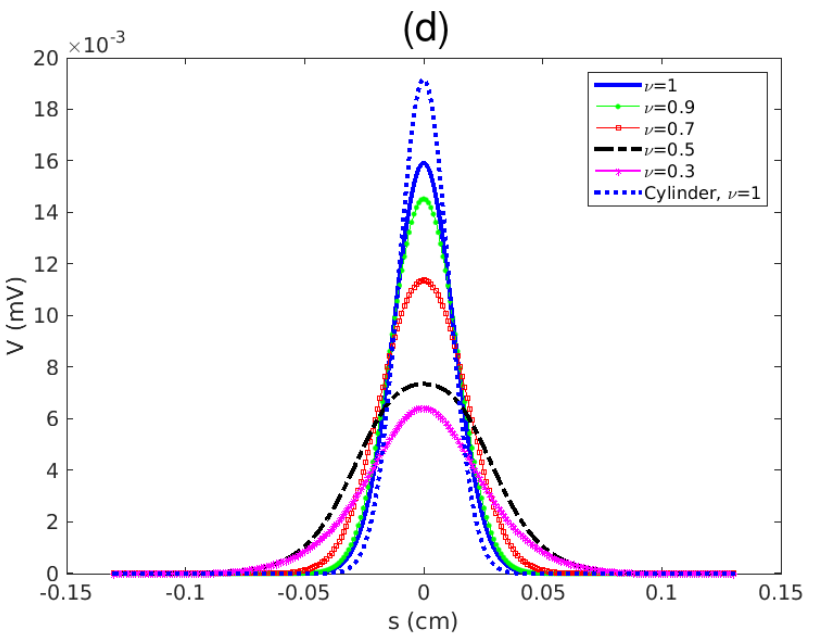

Figure 6: (a) Cable with geometry 422). (b) Voltage for the cable with the radius (42) and $\nu=0.5, \beta_{0.5}=16(\mathrm{sec})^{0.5}$. (c) Voltage vs $t$ in $s=0$ with different values of $\beta_{\nu}$ and $\nu$. (d) Voltage vs $s$ at time $t=7 \mathrm{sec}$ for different values of $\beta_{\nu}$ and $\nu$. Parameter values used for simulations correspond to realistic dendritic parameters as in [54]: $c_{M}=1 \mathrm{mF} / \mathrm{cm}^{2}, r_{M}=$ $3000 \Omega \mathrm{cm}^{2}, r_{L}=100 \Omega \mathrm{cm}, R_{0}=10^{-4} \mathrm{~cm}, \alpha_{1}=10, \alpha_{2}=0.11 \mathrm{~cm}^{-2}, \alpha_{3}=0 \mathrm{~cm}$. The initial condition is given by $(38)$. 
(a)

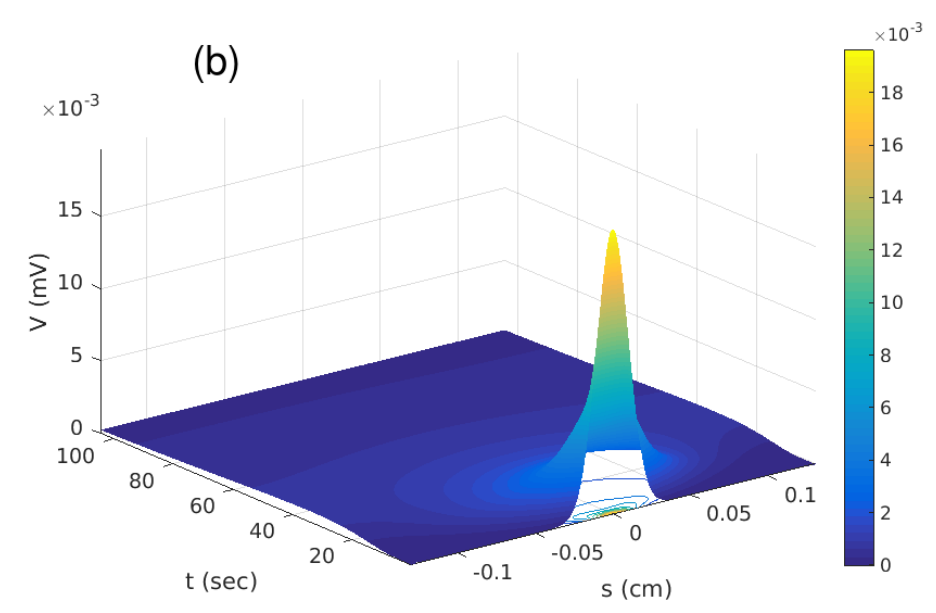

(c)
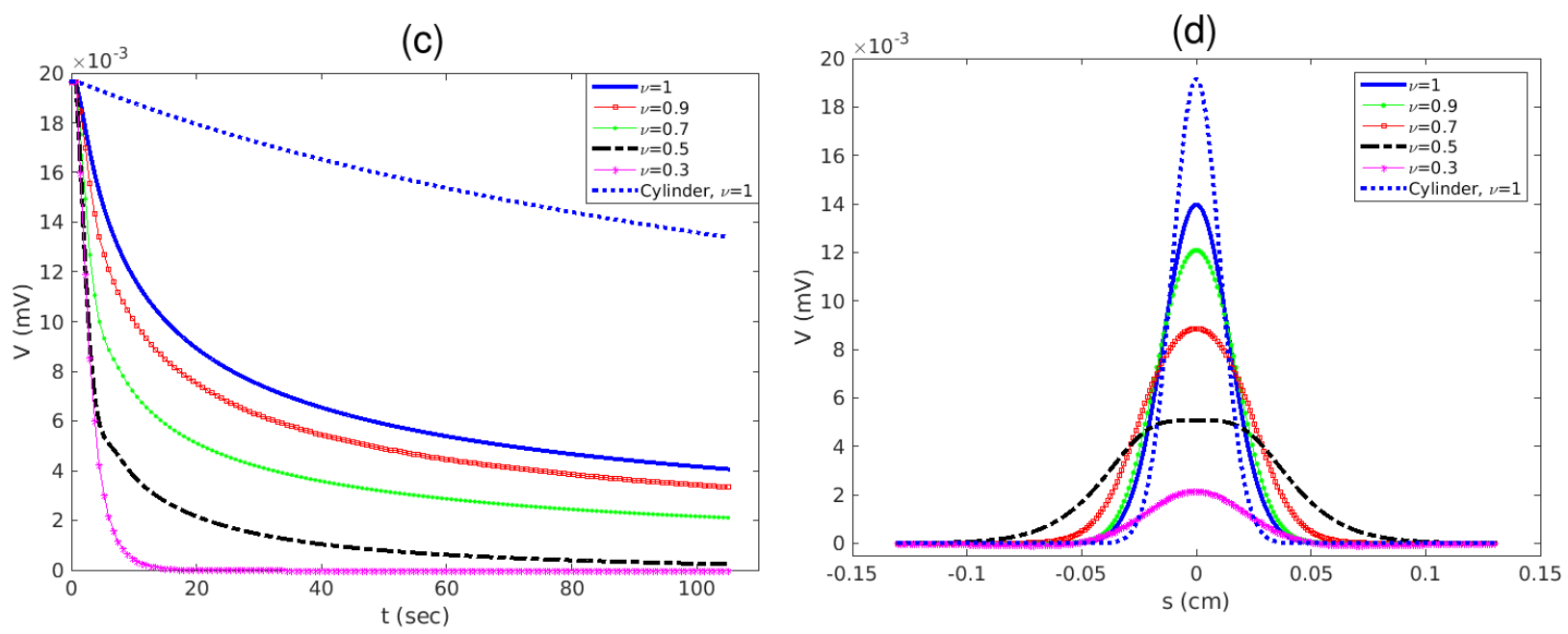

Figure 7: (a) Cable with geometry (43). (b) Voltage for the cable with a gaussian train swellings, radius (43) and $\nu=0.5, \beta_{0.5}=16(\mathrm{sec})^{0.5}$. (c) Voltage vs $t$ in $s=0$ with different values of $\beta_{\nu}$ and $\nu$. (d) Voltage vs $s$ at time $t=7 \mathrm{sec}$ for different values of $\beta_{\nu}$ and $\nu$. Parameter values used for simulations correspond to realistic dendritic parameters as in [54: $c_{M}=1 \mathrm{mF} / \mathrm{cm}^{2}, r_{M}=3000 \Omega \mathrm{cm}^{2}, r_{L}=100 \Omega \mathrm{cm}, R_{0}=10^{-4} \mathrm{~cm}, \alpha_{1}=10, \alpha_{2}=$ $0.11 \mathrm{~cm}^{-2}, \alpha_{3}=0.06 \mathrm{~cm}$. The initial condition is given by 38 . 
(a)

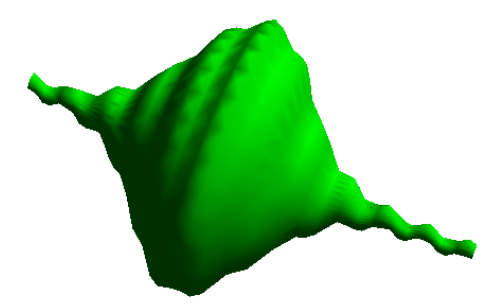

(b)
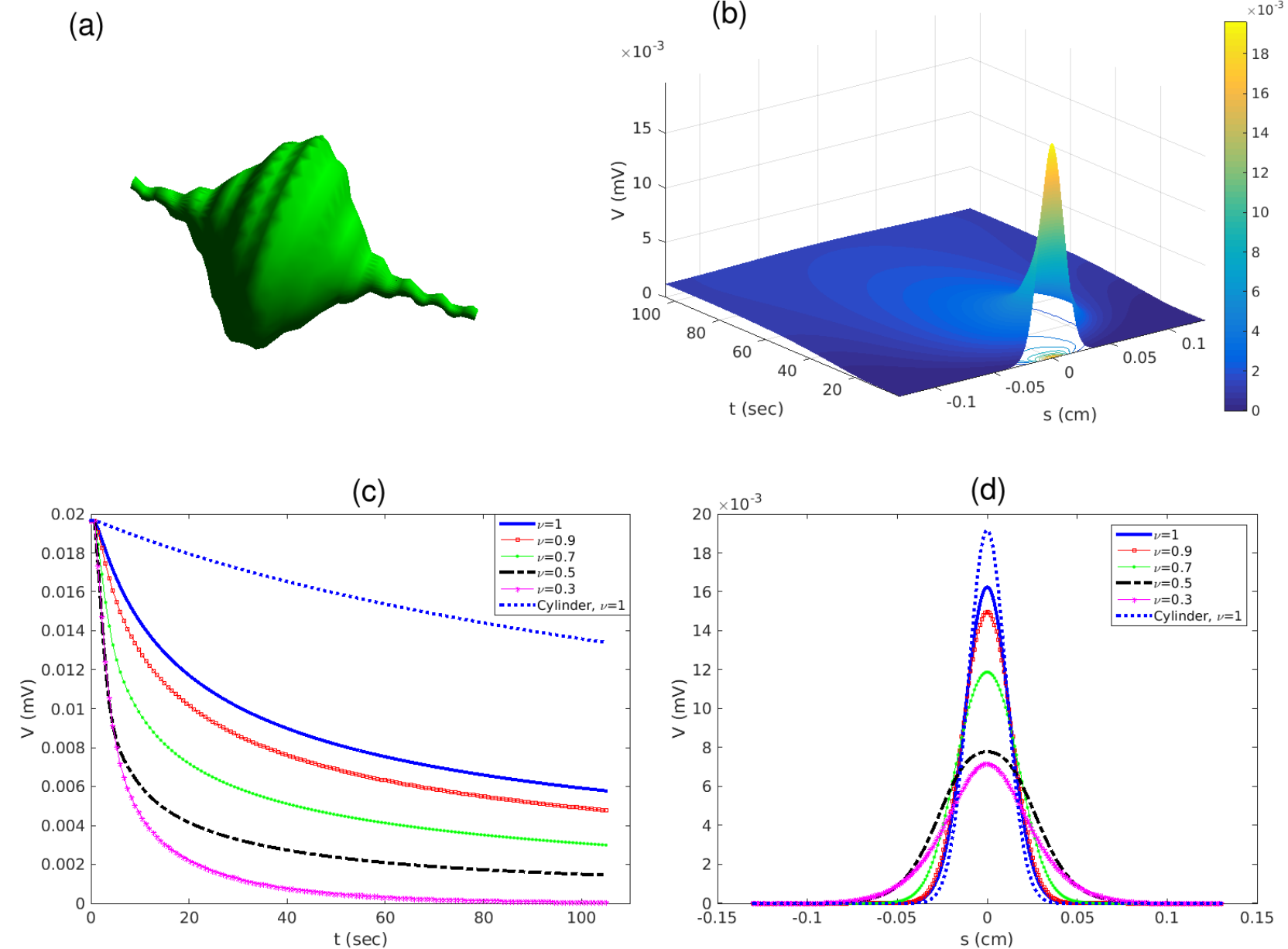

(d)

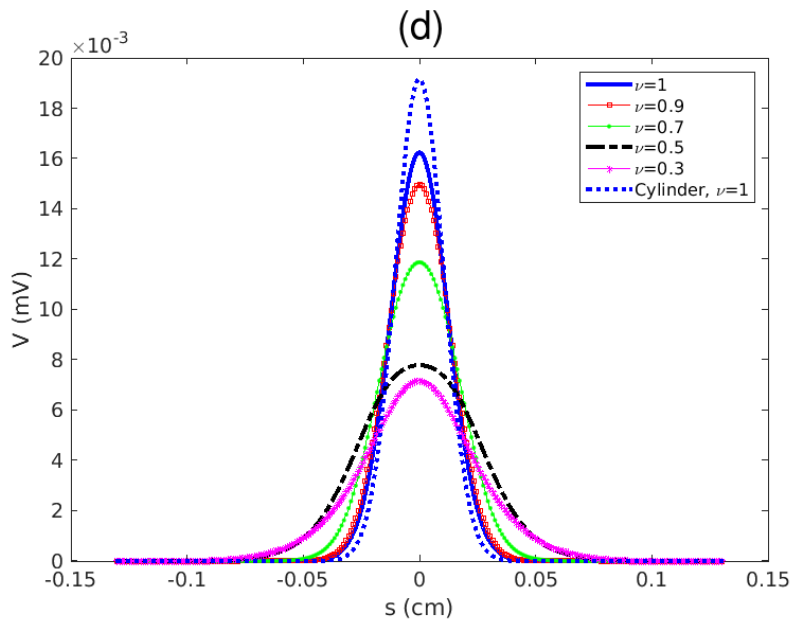

Figure 8: (a) Cable with geometry 44). (b) Voltage for the cable with the radius (44) and $\nu=0.5, \beta_{0.5}=16(\mathrm{sec})^{0.5}$. (c) Voltage vs $t$ in $s=0$ with different values of $\beta_{\nu}$ and $\nu$. (d) Voltage vs $s$ at time $t=7 \mathrm{sec}$ for different values of $\beta_{\nu}$ and $\nu$. Parameter values used for simulations correspond to realistic dendritic parameters as in [54]: $c_{M}=1 \mathrm{mF} / \mathrm{cm}^{2}, r_{M}=3000 \Omega \mathrm{cm}^{2}, r_{L}=$ $100 \Omega \mathrm{cm}, R_{0}=10^{-4} \mathrm{~cm}, \alpha_{1}=10, \alpha_{2}=0.11 \mathrm{~cm}^{-2}, \alpha_{3}=0 \mathrm{~cm}, \alpha_{4}=10, \alpha_{5}=0.11 \mathrm{~cm}^{-1}$. The initial condition is given by $(38)$. 